\title{
Das Bundesgericht präzisiert die Rechtsprechung zum Arztgeheimnis
}

\section{Ciro Papini}

MLaw, Rechtsdienst FMH

Das Arztgeheimnis steht im Fokus eines interessanten Bundesgerichtsurteils [1], bei dem die Bedeutung des Arztgeheimnisses und seine Ziele und Zwecke erläutert werden. Vorliegend werden die inhaltlich relevanten Aussagen des Bundesgerichts zusammengetragen.

\section{Die Ausgangssituation}

Der Kanton Tessin hatte eine Gesetzrevision durchgeführt, welche die Meldepflicht von Arztpersonen erheblich ausgeweitet und gleichzeitig deren Strafbarkeit im Falle einer unterlassenen Meldung verschärft hätte. So wurde u.a. Art. 68 Abs. 2 der Legge sulla promozione della salute e il coordinamento sanitario (Legge sanitaria, LSan, das kantonale Gesundheitsgesetz) insoweit abgeändert, dass Medizinalpersonen neu verpflichtet gewesen wären, innerhalb von $30 \mathrm{Ta}$ gen jede Krankheit, Verletzung und jeden Todesfall, die eindeutig oder vermutlich mit einem Offizialdelikt in Verbindung stehen, der Staatsanwaltschaft direkt oder via Kantonsarzt zu melden.

Zudem wurde Art. 68 LSan durch dessen Abs. 3 so ergänzt, dass Medizinalpersonen innerhalb von $30 \mathrm{Ta}$ gen dazu verpflichtet wurden, der Gesundheitsbehörde und der Staatsanwaltschaft jedes durch einen Kollegen oder eine Kollegin begangene Offizialdelikt

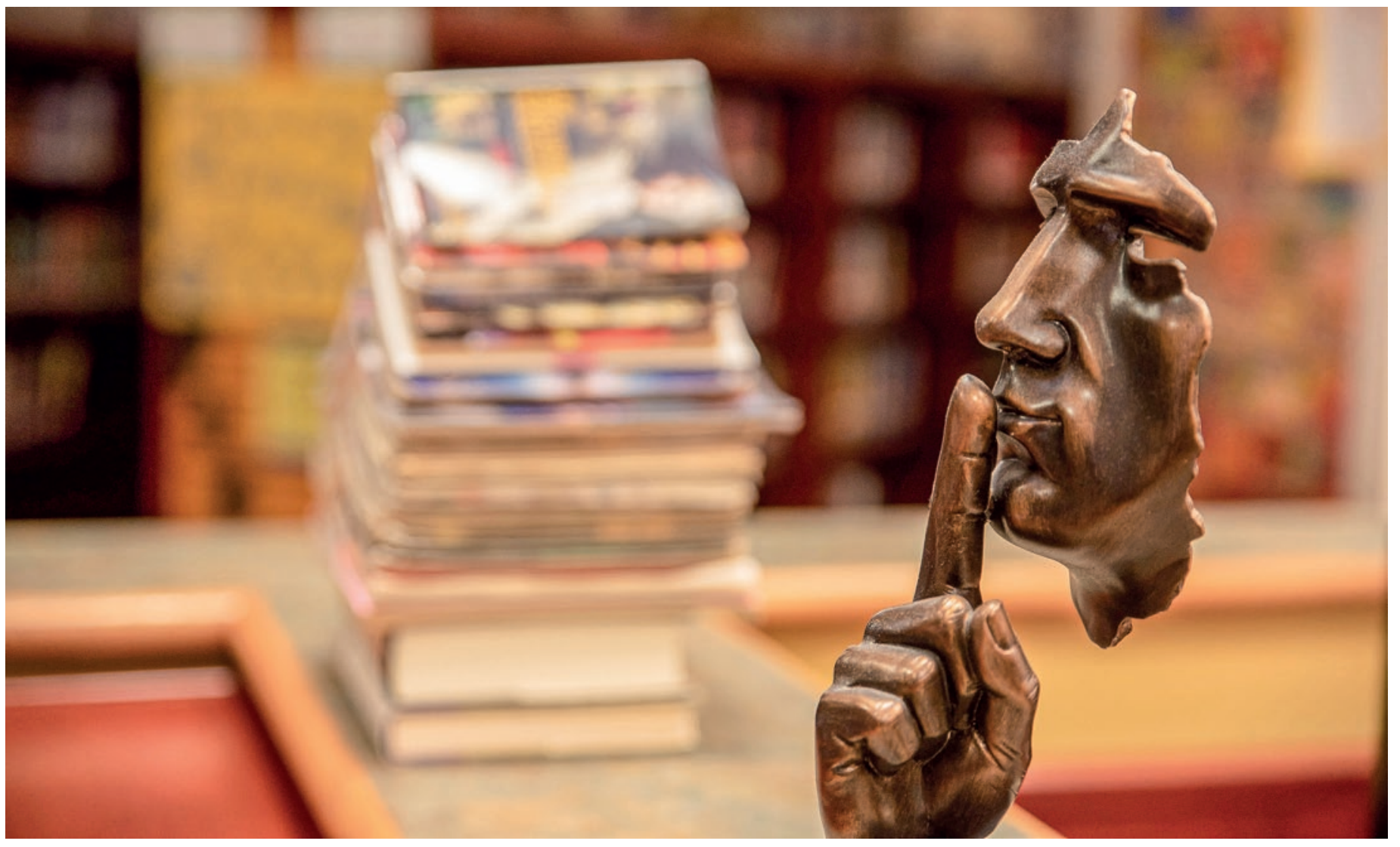


zu melden, welches im Rahmen deren medizinischen Tätigkeit begangen wurde.

Vier Tessiner Ärzte und Ärztinnen haben gegen diese Gesetzesänderungen Beschwerde beim Bundesgericht eingereicht und unter anderem verlangt, dass Art. 68 Abs. 2 und 3 LSan zu streichen sind.

Das Bundesgericht hat ihnen teilweise recht gegeben.

\section{Das Arztgeheimnis}

Das Bundesgericht hat im Urteil erneut klargestellt, dass das Arztgeheimnis ein zentrales und wichtiges Rechtsinstitut darstellt. Es dient der Verwirklichung des verfassungsmässigen Anspruchs auf Privatsphäre und schützt die Geheimsphäre des Patienten. Darüber hinaus dient es der öffentlichen Gesundheit, indem es ermöglicht, dass sich der Patient oder die Patientin dem Arzt oder der Ärztin ohne Vorbehalt anvertrauen kann und zweckmässig behandelt wird. Fehlt ein solcher Vertrauensschutz, könnten Personen, die eine

\section{Das Bundesgericht hat im Urteil erneut klarge-} stellt, dass das Arztgeheimnis ein zentrales und wichtiges Rechtsinstitut darstellt.

medizinische Versorgung benötigen, davon abgehalten werden, dem Arzt oder der Ärztin persönliche und intime Informationen bekanntzugeben, die für die Durchführung einer angemessenen Behandlung erforderlich sind. Oder sie könnten davon absehen, den Arzt zu konsultieren, was ihre eigene Gesundheit oder - im Falle von übertragbaren Krankheiten - die Öffentlichkeit gefährden könnte.

Das Arztgeheimnis gilt aber nicht absolut. Bund und Kantone dürfen es durch die Einführung von gesetzlichen Melde- oder Informationspflichten einschränken (Art. 321 Ziff. 3 StGB). Das Bundesgericht erinnert aber daran, dass im Grundsatz das Geheimnis gilt und in Bezug auf dessen Befreiung weniger weitreichende Lösungen bevorzugt werden sollten, wie z.B. die Entbindung vom Berufsgeheimnis durch den Patienten bzw. die Patientin und in zweiter Linie die Entbindung durch die Behörde. Die Einschränkung des Arztgeheimnisses durch die Einführung von Meldepflichten soll somit die Ausnahme darstellen.

Bei der Einführung von Art. 68 Abs. 2 LSan vermisste das Bundesgericht jedoch die für die Einführung von Meldepflichten nötige Interessenabwägung. Die infrage stehende Meldepflicht wäre nicht auf bestimmte Situationen beschränkt, in denen das Interesse an einer Offenlegung die Wahrung des Berufsgeheimnisses in jedem Fall überwiegt. Die Beschwerde wurde also in diesem Punkt gutgeheissen, und der Kanton wurde an- gewiesen, die Norm so zu formulieren bzw. einzuschränken, dass eine Meldepflicht «nur» bei Todesfällen bestehen darf, welche auf eindeutige oder vermutete Straftaten zurückzuführen sind. Die Ausweitung der Meldepflicht auf Verletzungen und Krankheiten ging dem Bundesgericht zu weit.

Diese Präzisierung des Bundesgerichts ist umso wichtiger, wenn man bedenkt, dass eine Verletzung des Arztgeheimnisses strafrechtlich verfolgt werden kann. Die problematischen Tatbestände müssen deswegen so klar wie möglich im Gesetz umschrieben sein, damit die angesprochenen Personen sich auch entsprechend verhalten können. Die Einführung einer Meldepflicht von jeglichen Straftaten, die durch Medizinalpersonen begangen werden und die von Amtes wegen verfolgt werden müssen (z.B. Steuerhinterziehung), wie der Kanton Tessin sie mit Art. 68 Abs. 3 LSan einführen wollte, ist zu pauschal, ermöglicht die nötigen Interessenabwägungen nicht und ist strafrechtlich betrachtet zu wenig präzis.

Aus diesem Grund hat das Bundesgericht den beschwerdeführenden Ärzten auch in Bezug auf diese Norm recht gegeben und Art. 68 Abs. 3 LSan aufgehoben.

\section{Die Abklärungspflicht}

Die Beschwerde richtete sich weiter gegen eine zusätzliche neu eingeführte Norm. Mit einem neuen Abs. 4 des Art. 20 LSan wollte der Kanton vorsehen, dass Medizinalpersonen sich nicht auf das Arztgeheimnis berufen können, falls die Aufsichtsbehörde von ihnen Informationen im Rahmen der Ausübung ihrer Aufsichtsfunktion verlangt. Obwohl das Bundesgericht die Beschwerde zu diesem Punkt abgewiesen hat, sind die Ausführungen dazu für die Praxis relevant [2].

Das Bundesgericht hat klargestellt, dass die Aufsichtsbehörde unter Wahrung des Verhältnismässigkeitsprinzips in der Regel in drei Situationen die notwendigen Informationen von Ärztinnen und Ärzten einfordern kann:

1. Im Rahmen ihrer gesetzlich vorgeschriebenen und regelmässigen Kontrollfunktion (Überprüfung der Dienstleistungsqualität alle 3-4 Jahre);

2. im Rahmen einer reaktiven Kontrolle (nach einer Meldung eines Patienten) oder

3. nach einer Meldung der Staatsanwaltschaft.

Im zweiten Fall nimmt das Bundesgericht an, dass das Einverständnis für die Akteneinsicht des Patienten oder der Patientin automatisch vorhanden ist. Der behandelnde Arzt darf sich somit nicht auf das Arztgeheimnis berufen. 
In den zwei anderen Konstellationen muss die Aufsichtsbehörde hingegen auch ohne die Einwilligung der Patientinnen und Patienten Zugang zu den nötigen Akten und somit, wenn im Einzelfall notwendig, auch zur Krankengeschichte haben, damit sie ihre gesetzlich vorgeschriebene Aufsichtspflicht wahrnehmen kann. Die Aufsichtsbehörde delegiert zudem in der Regel diese Kontrollaufgabe den Kantonsärztinnen. Diese sind einerseits selbst an das Amtsgeheimnis gebunden und andererseits ermächtigt, die infrage stehenden Ärztinnen und Ärzte vom Arztsgeheimnis zu befreien. Das Bundesgericht stellt somit klar, dass sich in solchen Fällen die betroffenen Ärztinnen und Ärzte nicht auf das Arztgeheimnis berufen können, um die Akteneinsicht der Aufsichtsbehörde zu verweigern.

\section{Konsequenzen für die Praxis}

Dieses Urteil ist insofern für die Ärzteschaft wichtig, als es nicht nur die Grundsätze in Verbindung mit dem Arztgeheimnis für die medizinische Praxis wiederholt und präzisiert, sondern auch klare Anweisungen an die Gesetzgeber erteilt, welche Anforderungen Ausnahmen vom Arztgeheimnis in Form von Meldepflichten erfüllen müssen.

Das Arztgeheimnis stellt für das Bundesgericht einen sehr wichtigen Bestandteil der Arzt-Patienten-Beziehung dar, und grundsätzlich muss davon ausgegangen werden, dass keine Informationen aus der Behandlung preisgegeben werden dürfen. Dieser Grundsatz ist in Art. 321 StGB festgehalten.

Die primäre Ausnahme von diesem Grundsatz ist die Einwilligung des Patienten bzw. der Patientin zur In- formationspreisgabe. Falls der Patient nicht kann oder will, soll wenn nötig die Befreiung vom Arztgeheimnis durch die Kantonsärztin oder den Kantonsarzt erfolgen (Art. 321 Abs. 3 StGB). Entscheidend dabei ist, dass im Einzelfall die notwendige Abwägung zwischen dem Interesse der Arztperson, eine geschützte Information preiszugeben, und dem Interesse des Patienten, die Information zu schützen, durchgeführt werden kann.

Die Aufhebung des Arztgeheimnisses durch die Einführung von gesetzlichen Meldepflichten muss die Ausnahme darstellen.

Die Aufhebung des Arztgeheimnisses durch die Einführung von gesetzlichen Meldepflichten muss sich hingegen stets auf sehr spezifische und klar eingrenzbare Sachverhalte beziehen und gleichzeitig die Ausnahme darstellen (Art. 321 Abs. 3 StGB). Dabei stellt das Bundesgericht fest, dass der Gesetzgeber im Lichte der oben genannten Grundsätze und unter Berücksichtigung der Wichtigkeit des Arztgeheimnisses bei der Einführung einer Meldepflicht eine Interessenabwägung zwischen den Interessen der Öffentlichkeit an einer Preisgabe einer sensiblen Information und dem Schutz des Vertrauensverhältnisses zwischen Arzt und Patientin durchführen muss.

\footnotetext{
Literatur

1 Urteil des Bundesgerichts 2C 658/2018 vom 18.3.2021.

2 Siehe Artikel «Wie reagiere ich auf Auskunftsbegehren der Aufsichtsbehörde?». Schweiz Ärzteztg. 2021;102(24):799-800.
}

Bildnachweis

Ernie A. Stephens / Unsplash 